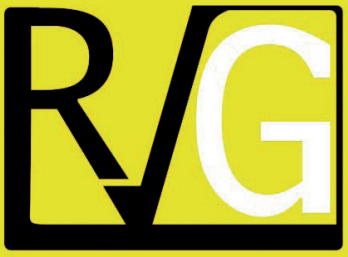

Julio - Septiembre, 2021

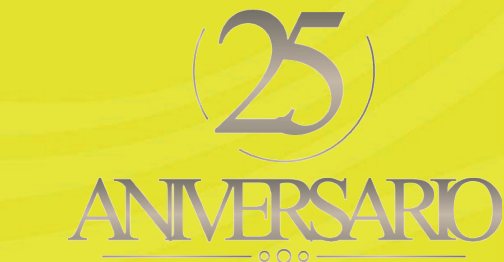

ANMERSARO
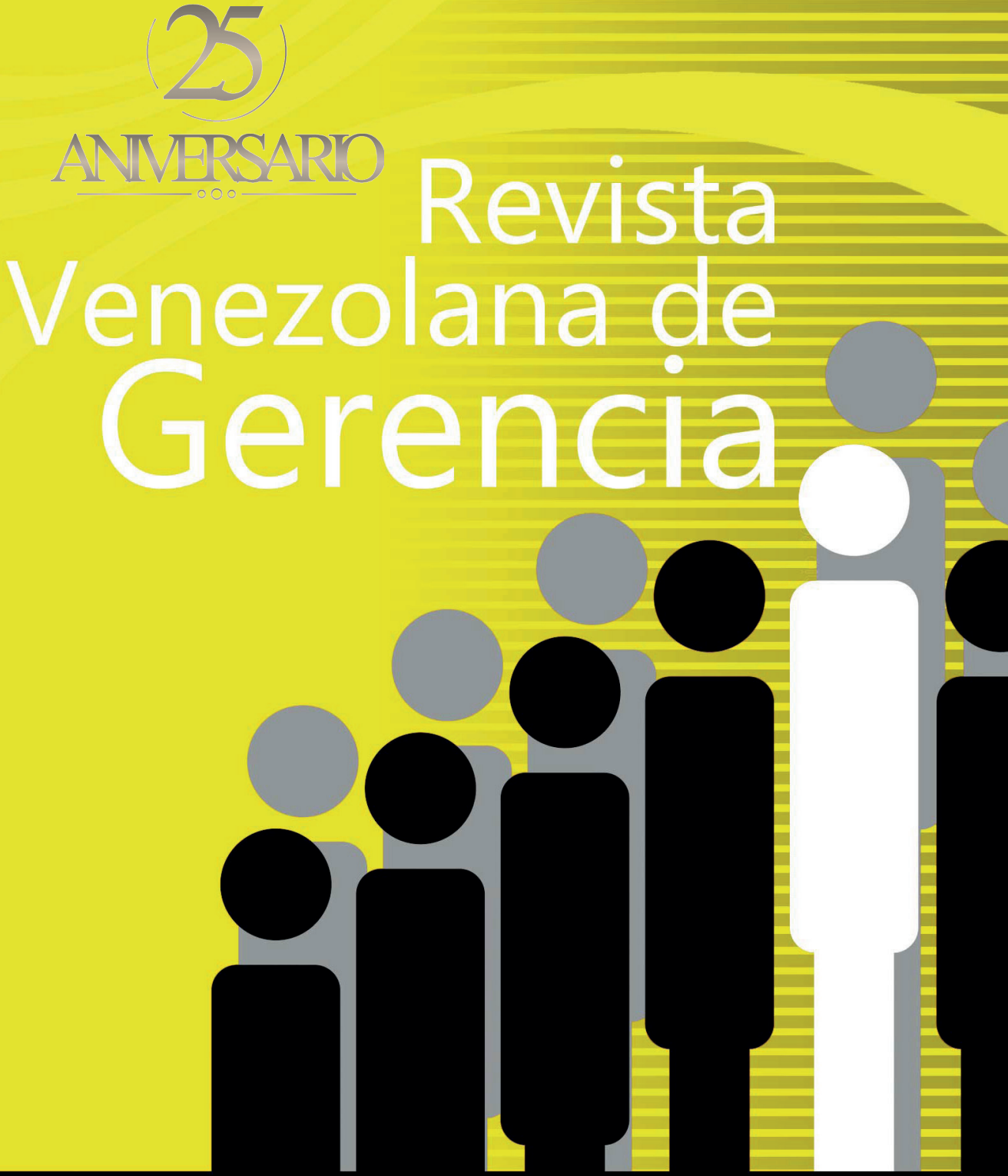

UNIVERSIDAD DEL ZULIA (LUZ)

Facultad de Ciencias Económicas y Sociales Centro de Estudios de la Empresa

ISSN 1315-99

Esta obra está bajo una licencia de Creative Comm Reconocimiento-NoComercial-Compartirlgual 3.0 Unpo http://creativecommons.org/licenses/by-nc-sa/3.0/deed.es 
COMO CITAR: Sánchez Montero, I. K., Ríos Mariño, M. J., Cajas Cajas, V. E., y Tanqueño Colcha, O. P. (2021). Liderazgo positivo en organizaciones saludables. Revista Venezolana de Gerencia (RVG), 26(95), 544563. https://doi.org/10.52080/

\author{
Universidad del Zulia (LUZ) \\ Revista Venezolana de Gerencia (RVG) \\ Año 26 No. 95 Julio-Septiembre 2021, 544-563 \\ ISSN 1315-9984 / e-ISSN 2477-9423
}

rvgluz.27.95.7

\title{
Liderazgo positivo en organizaciones saludables
}

\author{
Sánchez Montero, Ivanna Karina* \\ Ríos Mariño, Marcelo Javier* \\ Cajas Cajas, Viviana Elizabeth ${ }^{* * *}$ \\ Tanqueño Colcha, Oscar Paul ${ }^{\star \star \star *}$
}

\section{Resumen}

La investigación tiene como objetivo, determinar las implicaciones del liderazgo positivo dentro de organizaciones saludables, con la intencionalidad de ofrecer alternativas para la obtención de resultados satisfactorios y romper con esquemas de trabajos tradicionales. El enfoque de la investigación es cualitativo de tipo documental, apoyada en el método hermenéutico, empleando la técnica de la triangulación de fuentes para interpretar los datos recolectados. Los resultados reflejan, que el liderazgo positivo se convierte en una herramienta que integra responsabilidad con las emociones de los trabajadores, convirtiéndose en estrategia para que las organizaciones saludables emerjan como innovadoras y modelo a seguir. Otro resultado, es la creación de un sistema (SARF) que sirve de guía para el reconocimiento de los efectos positivos generados por el empleo de un programa basado en el liderazgo positivo. Las conclusiones apuntan a que el liderazgo positivo, genera en las organizaciones saludables un cambio paradigmático beneficioso comprobando que la unificación entre lo gerencial y lo emocional, abre espacios comunicacionales que enfocadas correctamente, generan escenarios estratégicos para tomar decisiones acertadas para el bienestar de todos los involucrados en la organización

Palabras clave: liderazgo positivo; organizaciones saludables; prácticas organizacionales; estrategias; innovación

* Máster Empresarial en Dirección Comercial y Marketing; Real Centro Universitario Escorial - María Cristina Adscrito a la Universidad Complutense de Madrid - España; Docente; Universidad Tecnológica Indoamérica - Ecuador; ivannasanchez@uti.edu.ec, http://orcid.org/0000-0002-0029-6265

** Magíster en Administración de Empresas y Marketing; Universidad Tecnológica Indoamérica - Ecuador; Docente; Universidad Tecnológica Indoamérica - Ecuador; marcelorios@uti.edu.ec, https://orcid.org/00000001-7634-0982

*** Magíster en Administración de Empresas y Marketing; Universidad Tecnológica Indoamérica - Ecuador; Docente; Universidad Técnica de Cotopaxi - Ecuador; viviana.cajas5044@utc.edu.ec, http://orcid.org/0000$\underline{0002-7688-6966}$

**** Magíster en Contabilidad y Auditoría; Universidad Nacional de Chimborazo - Ecuador; Docente; Universidad Estatal de Bolívar - Ecuador; otanqueno@ueb.edu.ec, https://orcid.org/0000-0002-5718-335X 


\title{
Positive leadership in healthy organizational
}

\begin{abstract}
The objective of the research is to determine the implications of positive leadership within healthy organizations, with the intention of offering alternatives for obtaining satisfactory results and breaking with traditional work schemes. The research approach is qualitative of the documentary type, supported by the hermeneutical method, using the technique of triangulation of sources to interpret the collected data. The results reflect that positive leadership becomes a tool that integrates responsibility with the emotions of the workers, becoming a strategy for healthy organizations to emerge as innovators and a role model. Another result is the creation of a system (SARF) that serves as a guide for the recognition of the positive effects generated by the use of a program based on positive leadership. The conclusions suggest that positive leadership generates a beneficial paradigmatic change in healthy organizations, proving that the unification between managerial and emotional issues opens communicational spaces that, when properly focused, generate strategic scenarios to make correct decisions for the wellbeing of all those involved. In the organization
\end{abstract}

Keywords: positive leadership; healthy organizations; organizational practices; strategies; innovation

\section{Introducción}

El análisis e interpretación sobre la conducta organizacional, ha permitido integrar componentes psicológicos y sociales en materia laboral (sinergia, empatía, entorno familiar, salud, entre otros) que antes no eran evaluados por las corporaciones, debido a que las metas de cumplimiento tenían un basamento focalizado hacia la productividad y rentabilidad (Reese, 2020). Señalan Cedeño et al. (2019) que, para mantener un comportamiento equilibrado y productivo dentro de las entidades, es importante que la alta gerencia mantenga la mirada hacia las realidades que circundan o forman parte del funcionamiento del negocio, sobre todo aquellas que generan cambios en las estrategias que pudieran afectar la toma de decisiones.

La existencia del desconocimiento, subrayan los autores, sobre situaciones internas o externas que afectan el ciclo normal operativo de la compañía (compras, ventas, finanzas), generan distorsiones e incertidumbres de manera directa a todo el entorno empresarial. Es lo que Gil et al. (2011) destacan como la permanencia de un estilo de liderazgo no adaptativo a la actualidad, desconociendo abiertamente las funciones y responsabilidades organizacionales cuyas consecuencias desencadenan desfases operativos, perdiéndose la trazabilidad de los objetivos planteados, la falta de conocimiento sobre la efectividad de los procesos y desapego de la calidad de 
sus resultados. Dicha situación podría convertirse en principal detonante de posibles vulnerabilidades, debacle y extinción de las empresas en el mundo de los negocios.

Es así como ha evolucionado de manera armónica un elemento gerencial ya conocido, pero esta vez, direccionado hacia conductas extraordinarias que producen cambios drásticos y efectivos en las organizaciones saludables. Nos referimos al liderazgo positivo, cuyo basamento se encuentra en la psicología y en la conducta organizacional; logrando engranar acciones y actitudes que potencian procesos vulnerables, producto de las complejidades $y$ disrupciones que padecen las entidades, bien sea, por factores competitivos o modernizaciones tecnológicas.

Es oportuno destacar, que las organizaciones saludables son aquellas que mantienen relaciones con toda la masa trabajadora, generando entornos competitivos acordes a cada nivel de responsabilidad del capital humano (Salanova, 2009). Es forjadora de puntos de equilibrios necesarios para producir oportunidades, que más tarde se convierten en productividad y competitividad. Entre las características principales, Salanova et al. (2016) mencionan que el establecimiento de emociones mediante el liderazgo positivo, influyen en el desarrollo de los trabajadores mientras se encuentran en el cumplimiento de las obligaciones. Esto se convierte en un elemento transformador de nuevos paradigmas gerenciales, ya que accede a combinarse con el conocimiento, competencia, experiencia con la salud (mental y física), emociones y calidad de vida.

El liderazgo positivo, ha sido reconocida como uno de los procesos de influencia social que más atención ha recabado en las ciencias conductuales $\mathrm{y}$, en particular, en la psicología organizacional; siendo la razón primordial para su estudio el éxito operativo desde el ámbito económico, político y organizacional, emanando una estrecha dependencia de la acción eficiente de los líderes de dichos sistemas (Vaca, 2019). Señala el autor, que el rol principal del liderazgo en el mundo de los negocios es crucial, debido a que empuja y promueve el bienestar y la salud laboral, a nivel individual y colectivo; posibilitando la aparición de diferentes modelos de liderazgo, como, por ejemplo, el positivo; cuya coincidencia está vinculada al modo en cómo los líderes estimulan y mantienen los niveles de rendimiento en sus colaboradores, por medio del fomento de comportamientos virtuosos apoyados en la felicidad.

Capa et al. (2020) plantean, que el liderazgo positivo, puede generar una fusión exitosa en dos organizaciones culturalmente diferentes, además de mejoras significativas en el desempeño financiero, aumentos notables en la satisfacción del cliente y un compromiso con los empleados. Su correlación con las organizaciones saludables, permiten darle un sentido humano a las relaciones gerenciales y comunicacionales, mediante la formación de líderes que no se enfoquen solamente en los resultados, sino que integralmente tengan la capacidad para combinar trabajo y emociones, sin perder de vista los objetivos empresariales establecidos. Cabe destacar, que las empresas enmarcadas bajo esquemas saludables, permiten combinar una serie de factores que propulsan la excelencia, fusionando ambientes laborales agradables, fortalecimiento de 
relaciones psicosociales, salud mental y física, apertura de vías comunicacionales y responsabilidad social; sin descuidar la misión y visión organizativa.

En este contexto, la presente investigación tiene como objetivo determinar las implicaciones del liderazgo positivo dentro de organizaciones saludables, con la intencionalidad de ofrecer alternativas para la obtención de resultados satisfactorios y romper con esquemas de trabajos tradicionales. El enfoque de la investigación es cualitativo de tipo documental, basado en la interpretación de las diferentes teorías y contextualizaciones sobre los principales ejes temáticos. Para ello, se apoya en el método hermenéutico, necesaria para interpretación tanto de las teorías como de los datos recolectados (la entrevista a informantes claves). Su naturaleza tiene carácter inductivo, holístico y heurístico; así como, abierto y flexible, características destacables para desarrollar la capacidad que tiene el hecho observable en torno a las realidades que conforman al estudio.

La técnica utilizada para la recolección de la información fue la revisión de documentos, complementada con la realización de entrevistas a profundidad a tres informantes clave catalogados como expertos según preparación, competencias, experiencia profesional en los ejes temáticos principales que permiten dilucidar y soportar las categorías (previas y emergentes) que surgen del análisis del instrumento (matriz triangular) también conocido como material protocolar.

\section{Liderazgo positivo herramienta frente al cambio}

Precisa Benavides (2017), que las organizaciones laboran bajo entornos dinámicos y crecientes, engendrando elementos como la incertidumbre que a pesar de que no se puede eliminar, si se logra controlar. Enfatiza el autor que, para disminuir este efecto propio de algunas corporaciones, es necesario la aparición de líderes con cierto grado de independencia y pensamientos distintos que se atrevan a romper paradigmas y generen sinergias emergentes a todos los que integran la empresa, con miras a mejorar los niveles productivos, armónicos, innovadores, creativos y competitivos; para la obtención de metas establecidas.

En ese sentido, el liderazgo positivo es reconocido como una herramienta de destreza estratégica a ser tomada en consideración en la concavidad de las organizaciones, debido a que los resultados operativos que emana tiene incidencia significativa en la productividad y competitividad, fortaleciendo a su vez, vínculos afectivos entre los involucrados (mano de obra) en el proceso empresarial (Díaz, 2017).

Señala Cameron (2013), que su fundamento se encuentra en la aplicación de principios de conducta que emergen de disciplinas como la psicología positiva y la psicología organizacional positiva. Asimismo, plantea que el liderazgo positivo tiene tres componentes básicos: a) posiciona el centro de interés en aquellas fortalezas y habilidades de las personas que reafirman su potencial humano; b) pone el énfasis en los resultados y facilita un rendimiento individual y organizacional por encima del promedio; y c) su campo de actuación se concentra en aquellos componentes que pueden concebirse como virtudes esenciales de la condición humana. A juicio de Belanger et al. 
(2015) las estrategias derivadas de esta visión, coadyuvan a que los equipos de trabajo alcancen efectividad y, a su vez, una productividad que sobrepasa los estándares gerenciales.

No obstante, para que el liderazgo positivo sea beneficioso, Chiniara \& Bentein (2016) expresan la necesidad de que los líderes instauren ambientes de trabajo extremadamente competentes. La idea es lograr el éxito bajo este esquema estratégico, que refuerza los puntos fuertes (competencias) que posee de cada integrante de la organización, en lugar de concentrarse en las metas individuales. Con relación a lo anterior, Díaz (2017) sugiere que los liderazgos empresariales modernos, deben enfocarse en la distinción de aquellos puntos álgidos que poseen intrínsecamente los trabajadores, con el propósito de brindarles seguridad y confianza, aprovechando los recursos en cuanto a capacidad y aptitudes de las personas.

Ahora bien, convertirse en un verdadero líder para Goodall \& Pogrebna (2014), conlleva reunir ciertas aptitudes que ocasionan nexos de confianza necesarios para promover emociones positivas, dicho de otra manera, debe desarrollar interacciones de apoyo mutuo sin ningún tipo de discriminación, con el objeto de transmitirle al equipo de trabajo, el verdadero valor y propósito de las tareas. Investigaciones recientes como las de Capa et al. (2020); Guerrero et al. (2018); Roche et al. (2017); y Salanova et al. (2016) revelan que cuando se crea un clima favorable en el ámbito laboral, se crean al mismo tiempo la optimización y logros de alto desempeño por encima del promedio que la alta gerencia espera.

Por su parte, Donoso y Benavides
(2018) proponen unas características que debe poseer un líder, figurando: la empatía, comprensión, atención, precisión, comunicación, carisma, espíritu innovador, compromiso, efectividad y credibilidad.Adicionalmente, competencias axiológicas (valores) para lograr confianza y admiración por el equipo de subordinados, en otras palabras, debe tener la capacidad para escuchar a todos los miembros y lograr engranar y fijar una posición grupal, con miras a obtener alternativas y ofrecer la mejor opción hacia la toma de decisión.

Así pues, los líderes se convierten en los encargados de mantener un ambiente organizacional que permita el crecimiento y aprovechamiento de emociones positivas (alegría, confianza, amor, apreciación) y disminuya las negativas (miedo, rabia, ansiedad, inseguridad) provocando un crecimiento notable que desarrolla capacidades cognitivas respaldando elementos estratégicos (creatividad, innovación y proactividad), que permiten la seguridad individual y colectiva fortaleciendo el desempeño laboral. Lo cual a su vez genera la capacidad organizacional para hacer frente a los cambios veloces y vertiginosos que afectan el mundo de los negocios.

Sobre este último aspecto, Rocha (2019) considera que el liderazgo supone la capacidad de persuadir a personas con el fin de lograr metas y objetivos bajo esquemas eficientes y efectivos. A ello, agregan Capa et al. (2020), detectar personas que trabajen por vocación y convertirlos en activo intangible para la organización. Lo anterior, mediante, la coordinación y consolidación del grupo al que representa, con el objetivo de fomentar una cultura del cambio y mejorar las realidades que circundan 
los negocios (Baño, 2019). En opinión de González (2019) la existencia de liderazgos que logran un impacto positivo en las entidades, destacan los que se rigen por la visión y misión empresarial focalizados en los objetivos. Para tener éxito en este sentido, es necesario diseñar estrategias que promuevan e identifiquen nuevas oportunidades, logrando motivar e incentivar al personal para que empleen su potencial innovador y desarrollen las capacidades que generen soluciones inteligentes y positivas frente al cambio.

Es mediante el liderazgo, que se puede lograr un consenso grupal que sirve de sustento para presentar escenarios múltiples o alternativos, y poder tomar decisiones acertadas apoyadas en conductas creativas que fortalecen a la organización. Es mediante el accionar del liderazgo positivo, que se pueden lograr cambios relevantes y significativos con la participación efectiva del pilar principal de una entidad como lo es la gente. Este tipo de herramienta es contagiosa, y permite realizar planificaciones en las conductas y actitudes de todos los involucrados en el proceso.

\section{Organizaciones saludables y buenas prácticas}

Las organizaciones saludables, de acuerdo con Salanova (2008), refieren un novedoso concepto, que refresca los enfoques relacionados con la relevancia del recurso humano empresarial, pero desde el plano productivo y de la salud, que juntos y armonizados de manera adecuada, refuerzan efectivamente procesos de la renovación y mejora continua dentro de las actividades que forman parte de la operatividad y rentabilidad del negocio.

Partiendo de lo anterior, se agrega que este nuevo matiz fundamentado ya no solo en la productividad, efectividad y eficiencia, sino en el contexto de lo conocido como saludable y cuidado de la masa trabajadora; añade elementos frescos e innovadores que tiene que ver con competencias, liderazgo, desarrollo profesional y psicosocial hacia la salud, que resultan de consideración para conseguir otros fines empresariales que aportan un nuevo estereotipo de valor estratégico, centrado hacia las metas y objetivos corporativos.

Contrastando algunas perspectivas teóricas, Solares et al. (2016) piensan que las organizaciones saludables son una tendencia en torno al valor estratégico de la compañía. Dicho valor, va acompañado de sistemas colaborativos que se delinean para maximizar bienestar general (salud) y productividad, mediante la estructuración idónea de responsabilidades acordes a las competencias del trabajador y la ergonomía laboral. En concordancia, a juicio de Coronel y Marzo (2017), existen elementos influyentes en las organizaciones saludables donde se destacan casualmente la salud y el ambiente laboral.

Para estos últimos autores, dichos elementos deben ser valorados por las organizaciones debido al valor intangible que representa al momento de evaluar eficiencia y efectividad laboral. Esta situación permite desarrollar positivamente la productividad, motivación y la calidad desde todos los puntos de vistas que envuelve la responsabilidad del trabajador. Reafirman que la búsqueda de la equidad empresarial donde se involucre la salud, la ergonomía laboral y los beneficios 
empresariales (utilidades); generan estructuras sólidas que, armonizadas adecuadamente, se transforman en calidad de vida para el trabajador.

En opinión de Wilson et al. (2004, p.567) "las organizaciones saludables son aquellas caracterizadas por invertir esfuerzos de colaboración, sistemáticos e intencionales para maximizar el bienestar de los empleados y la productividad, mediante la generación de puestos bien diseñados y significativos". La posición de los autores se encamina hacia la conformación de climas sociales que fundamenten el desarrollo de la efectividad operacional, siendo el liderazgo una de las herramientas que entran en escena para organizar y emprender procesos de integración y dirección de los trabajadores.

A partir de estas
contextualizaciones, se puede afirmar que, las organizaciones saludables son aquellas que poseen trabajadores y resultados saludables, obtenidos por medio de la estructuración de esfuerzos sistemáticos, planificados y proactivos, conel propósito de realizarcombinaciones de mejoras fundamentadas en la salud de los empleados y la salud financiera de la organización. Esa visión vertical y tradicional que considera a la masa trabajadora como instrumentos para conseguir los fines empresariales, viene a ser reemplazada por elementos atados a la psicología organizacional positiva que medita sobre la salud del trabajador, el cual debería ser visto como un objetivo estratégico, estudiando la posibilidad de incluirse como una política organizacional. Si se le busca un raciocinio científico a este concepto, es evidente que su enfoque y direccionalidad se enmarca hacia la generación de beneficios económicos y sociales, los cuales se encuentran por encima de las entidades conocidas como enfermas o tóxicas.

En virtud de solidificar a las organizaciones saludables, existen las buenas prácticas empresariales saludables cuyos principios se relacionan con la promoción y optimización de recursos que tienen que ver con la mejora de las tareas, el ambiente social y la empresa. Es bueno destacar, que las prácticas no son técnicas para desarrollar el potencial humano, más bien son buenas prácticas entendibles sobre estrategias para el colectivo, es decir, son componentes culturales intrínsecos de la empresa que deberían formar parte de la filosofía del negocio.

Así, las prácticas organizacionales saludables, constituyen un elemento que cada vez se hace indispensable integrar en los procesos productivos (Sena, 2016). En este sentido, la autora confirma que la mixtura actúa como clave esencial, ya que su constitución parte del fortalecimiento de los recursos laborales (recursos de tarea y sociales), así como, por prácticas organizacionales que se desarrollan desde la Dirección de Recursos Humanos y que tienen como norte; incrementar la salud psicológica positiva y financiera del capital humano. Esto se produce debido al modernismo, que se han dado la tarea de contratar trabajadores sanos y motivados con potencial para ejercer funciones bajo presión y que, al mismo tiempo, mediante el nivel de conocimiento y experiencia, expresen capacidad de respuestas ante cualquier tipo de adversidad, caos o complejidad.

Lo anterior es reafirmado por Acosta et al. (2013) cuando comentan que los empleados representan el activo más valioso dentro de la estructura 
organizativa, el cual mientras más tiempo se encuentren o formen parte en el equipo de trabajo, mayor cohesión y mejores resultados se presentaran a corto y mediano plazo. Adicionalmente, confirman que las prácticas saludables como estrategia, permiten la decisión sobre la permanencia del trabajador siempre y cuando se tomen en consideración medios saludables (puesto de trabajo) que conducen a lo siguiente: a) centrarse en el bienestar general del trabajador y todos los miembros del equipo que producen eficacia y desarrollo competitivo, y b) poder distinguir entre organizaciones saludables y no saludables, dicho de otra manera, diseñar una estructura basada en la gestión de los procesos internos y externos relacionados con las responsabilidades del trabajador que genera mejores resultados y competitividad empresarial. Hasta aquí

Adicional a lo señalado, una investigación realizada por Solares et al. (2016), denota una marcada diferencia sobre el diseño de las compañías bajo esquemas de buenas prácticas empresariales saludables, que involucran a todo el equipo de trabajo, previamente haciéndoles entender hacia donde se dirige la organización y cuál es su responsabilidad, dependiendo su competencia y sentido. Prácticamente, se genera un efecto de bienestar mutuo (sinergia) que activa procesos colectivos, es decir, a medida que el trabajador o el grupo de trabajo presenta resultados satisfactorios, de la misma forma deben sentir el respaldo emocional que la empresa ofrece. Mucho se habla acerca de los sueldos psicológicos, sin embargo, hay momentos donde lo tangible debe hacerse presente $y$, la masa trabajadora, necesita sentir que crece proporcionalmente con la organización.

A todas estas, así como existen esquemas o diseños para el empleo de prácticas relacionadas con las organizaciones saludables, también hay recursos que se deben evaluar, si de verdad se quieren potenciar ciertos aspectos del proceso integral de la empresa (ser diferentes). El propósito fundamental, es la optimización del bienestar y crecimiento del rendimiento laboral, sin perder de vista hacia donde se direcciona la compañía (misión y visión). Con relación a esto, Salanova (2009) detalla unos recursos diseñados para fortalecer las actividades intrínsecas o responsabilidades diferenciadoras de cada equipo de trabajo, bajo lineamientos organizacionales, comunicacionales, de responsabilidad laboral y diseño y rediseño de tareas. Los organizacionales, permiten desarrollar no solamente trabajos efectivos, sino también, el crecimiento de una carrera profesional de cualquier trabajador; los comunicacionales, promueven la conexión de la masa trabajadora bajo estrategias basadas en la selección de los trabajadores, formación, aprendizaje y desarrollo de competencias (empleabilidad), políticas de empleo estable y estrategias de conciliación vida laboral-vida privada.

Por su parte, los de responsabilidad laboral, refiere a aspectos como la claridad en las especificaciones de las tareas, dejando claro la importancia de la información, la autonomía para elaborar tareas y la elaboración de un esquema comunicacional que sirva para abrir canales de información y que sea revisada y discutida antes de tomar decisiones que afecten las directrices organizacionales. Finalmente, las de 
Sánchez Montero, Ivanna Karina; Ríos Mariño, Marcelo Javier;

Cajas Cajas, Viviana Elizabeth y Tanqueño Colcha, Oscar Paul

Liderazgo positivo en organizaciones saludables

diseño y rediseño de tareas, permite la retroalimentación de los procesos internos y externos. Se debe tener en cuenta las condiciones del trabajador, su capacidad, entorno donde elabora la información y sobre todo si contienen las herramientas y el adiestramiento necesario para ejecutar la tarea encomendada.

Esto, complementa las necesidades que tienen las organizaciones saludables para emplear estrategias que incluya lo conocido como las buenas prácticas, sobre todo por los constantes cambios que sufren producto de la utilización exhaustiva de la tecnología, la globalización y la creciente competencia; el cual conlleva a contar con personal capacitado bajo ciertas competencias que conduzcan a la obtención del máximo rendimiento en su puesto de trabajo. Como se puede notar no todo es conocimiento y experiencia, también debe existir un equilibrio que involucre motivación, psicología positiva, salud y generación de felicidad para que los resultados sean beneficiosos para ambas partes (ganar-ganar).

\section{Factores influyentes en el liderazgo positivo y las buenas prácticas en organizaciones saludables}

Para que existan diferencias marcadas a nivel organizacional, y se comience a escalar espacios basados en ventajas competitivas, se necesita, emprender procesos de cambio diferenciadores que no puedan ser imitados fácilmente por la competencia. Uno de esos cambios lo constituye el liderazgo positivo, fusionado con las buenas prácticas en organizaciones saludables. En ese sentido, uno de los propósitos de un sistema basado en un liderazgo positivo, radica en concretar el desarrollo de recursos humanos que vayan más allá que diseñar conjuntos específicos de programas y prácticas, que funcionen para todas las dependencias organizaciones por igual; $\mathrm{y}$, reproduzca automáticamente una espiral hacia arriba para construir y mantener la ventaja competitiva.

Ahora bien, existen factores que inciden en las empresas saludables y el liderazgo positivo, destacando cuatro: ambiente físico de trabajo, relaciones psicológicas de trabajo, participación de la empresa en la comunidad, y recursos individuales de salud en el trabajo. A continuación, se muestran en el diagrama 1, cada uno de estos factores considerados suficientes para que las organizaciones saludables obtengan resultados satisfactorios, apoyados en elementos referidos al liderazgo positivo. 


\section{Diagrama 1 \\ Factores que inciden en las prácticas y resultados de las organizaciones saludables}

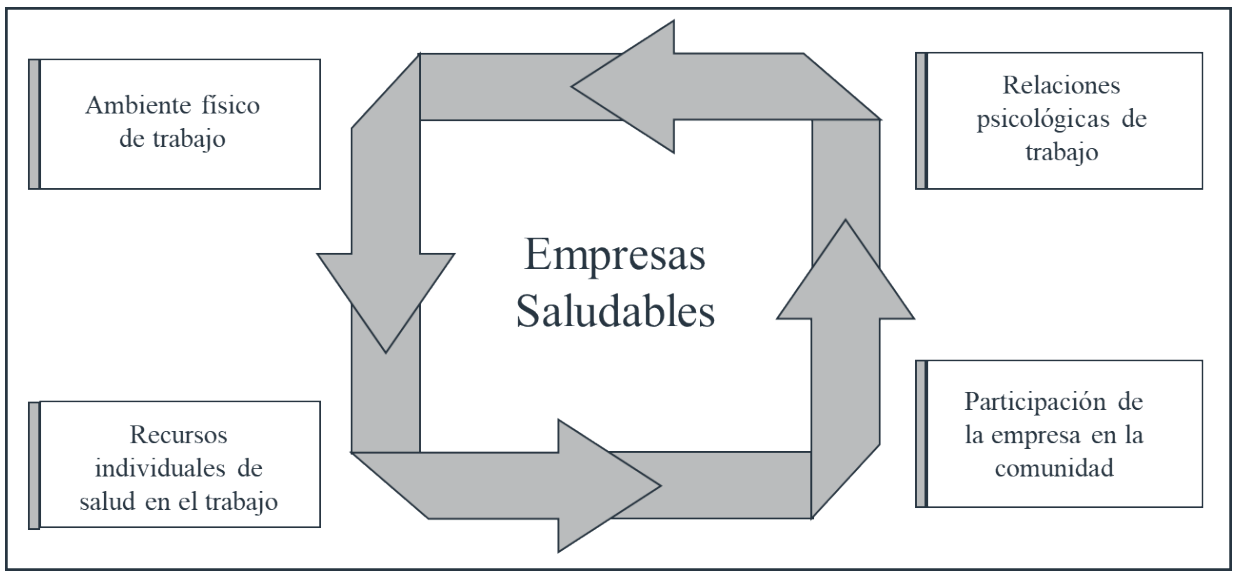

Fuente: David \& Quintero (2016)

El primer factor o ambiente físico de trabajo, además de proporcionar beneficios al personal, también genera productividad, competitividad y sostenibilidad, necesarias para desarrollar entornos para las economías globales. Villavicencio (2017) menciona, que un ambiente de trabajo saludable es aquel donde los trabajadores y las organizaciones se unifican para activar procesos enmarcados en la mejora continua para proteger la salud, seguridad y el bienestar de todos los empleados y la sostenibilidad del lugar de trabajo.

La Organización Internacional del Trabajo (OIT, 2008) mediante la Declaración de Seúl sobre seguridad y salud en el trabajo, deja claro que el ámbito laboral saludable debe ser visto como un derecho humano elemental. La relevancia de la declaración radica en plantar un liderazgo positivo que sea espontáneo y voluntario para el personal en general, reconociendo la presencia de normas y tratados sobre principios universales relacionados con los derechos humanos, las normas laborales, el medio ambiente y la lucha contra la corrupción.

El segundo factor o relaciones psicosociales de trabajo, se refiere según Rico (2016) a las condiciones presentes en una situación laboral y que están directamente relacionadas con la organización, el contenido del trabajo y la realización de las actividades; que afectan al bienestar y la salud (física, psíquica o social) del trabajador, tales como calidad de la comunicación y de las relaciones humanas Cuando se presentan condiciones psicosociales desfavorables, comienzan a aparecer diferentes conductas y actitudes inadecuadas que resultan perjudiciales para la salud y bienestar del trabajador. 
Estas consecuencias pudieran derivarse por: estrés, cansancio y fatiga mental, insatisfacción laboral, problemas de relación y desmotivación.

Añade la autora, que ciertas características de este factor inciden en el trabajador dependiendo de su personalidad, necesidades, expectativas, vulnerabilidad y capacidad de adaptación; de eta manera la naturaleza organizacional estará afectada por el factor psicosocial. Todo esto confirma, que son interacciones entre el trabajo y el entorno; aunado a las condiciones de la empresa las que permiten medir las verdaderas capacidades del trabajador, sus necesidades y su situación personal fuera del ambiente de trabajo, para poder obtener verdaderos niveles de rendimiento y efectividad laboral.

El tercer factor, es la participación de la empresa en la comunidad, donde se fusiona un tridente (empleadortrabajador-sociedad) que conlleva a diseñar estrategias hacia la creación y fortalecimiento de la seguridad social organizacional. Cuando se logra integrar crecimiento y socialización, se pueden conseguir innumerables resultados que conducen a una administración exitosa. Las organizaciones deben tomar en consideración aspectos sociales con la participación activa del personal, de forma tal que se logre la sensibilización colectiva. En este sentido, Sena (2016) sugiere que, para cubrir este punto, es importante tener control y un análisis exhaustivo de las condiciones externa donde se desenvuelve la empresa, con el fin de conocer qué proyectos podrirán ser factibles y necesario.

Finalmente, el cuarto factor, referido a los recursos individuales de salud en el trabajo, está relacionado con la atención a recursos que facilita el bienestar de los empleados, como es el caso de horarios flexibles, trabajo remoto, seguridad social, entre otros. En este sentido, Thomas (2017) resalta el empleo de instrumentos gerenciales, permite evaluar si una entidad realmente es efectiva empleando técnicas saludables organizacionales. Precisa el autor, que no todas las empresas pueden tener éxito en este tipo de recursos, sin embargo, aplicar métodos creíbles y probables conlleva a enfrentar cambios significativos que dan apertura a un nuevo éxito gerencial. Entre los efectos positivos de este factor está: la disminución de los accidentes y, el absentismo laboral, y la rotación de personal. Esto significa, que mejorando la salud, motivación y la participación integral; se logra confeccionar un adecuado clima laboral que al mismo tiempo mejora la imagen corporativa.

\section{Liderazgo positivo y organizaciones saludables: La visión de lo expertos}

Partiendo de los resultados y análisis arrojados por la matriz triangular empleada en la investigación, basada en las entrevistas a profundidad realizada a los informantes clave participantes, el estudio encontró tres categorías principales: liderazgo positivo, organizaciones saludables y responsabilidad social (diagrama 2). Respecto a esta última categoría, se compone de aspectos como: conciencia social, empatía, orientación al servicio y compromiso social. Se trata de una categoría emergente, no prevista en la investigación, que surgió de la información recabada (Silva \& Bonilla, 2020).

La síntesis conceptual de los resultados conlleva a afirmar, que un 
liderazgo positivo efectivo es el que obtiene capacidades de desarrollo del equipo de trabajo subordinado, empleando elementos persuasivos que permitan utilizar el tacto y otras habilidades sociales para estimularlos a lograr lo mejor (Bonilla, Mora y Macero, 2018). No se refiere a parecerse simpático o comprensivo, más bien a implementar acciones motivadoras individuales y grupales, en búsqueda de la direccionalidad para concretar beneficios efectivos y eficientes en pro de promover el objetivo de toda una organización.

\section{Diagrama 2 \\ Categorías y Subcategorías resultantes de la triangulación}
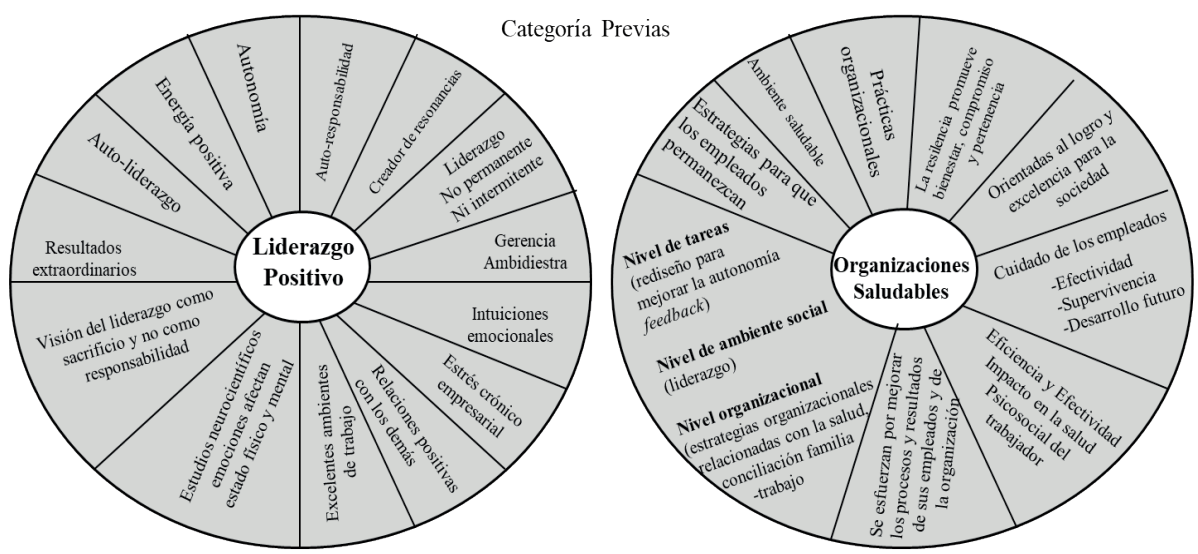

Categoría Emergente

\section{Responsabilidad Social}

\begin{tabular}{|c|c||c|c|c|c|}
\hline $\begin{array}{c}\text { Conciencia } \\
\text { Social }\end{array}$ & Empatía & $\begin{array}{c}\text { Conciencia } \\
\text { organizacional }\end{array}$ & $\begin{array}{c}\text { Orientación } \\
\text { al } \\
\text { servicio }\end{array}$ & $\begin{array}{c}\text { Compromiso Social } \\
\text { como estrategia } \\
\text { Intrínseca de la } \\
\text { gestión empresarial }\end{array}$ & $\begin{array}{c}\text { Conjugar } \\
\text { competitividad con } \\
\text { el compromiso } \\
\text { social }\end{array}$ \\
\hline
\end{tabular}

Fuente: Fuster (2020)

Con respecto a la categoría liderazgo positivo, las opiniones de los expertos sostienen que pretende el logro de una meta mediante la dirección de colaboradores humanos, pero fusionando responsabilidad y gerencia ambidiestra, es decir, integrando trabajo y bienestar (Bonilla, Mora y Macero, 2018). El hombre por su naturaleza es creador de resonancias que se integran a procesos transmisores de energía positiva, con miras a alcanzar fines comunes. No obstante, existe un componente que resulta lo 
suficientemente relevante como lo es la autonomía, que tiene que ver con las auto-dependencia que tiene un líder para ejercer roles que empujen todas aquellas intuiciones emocionales, tratando de consolidar auto-responsabilidad grupal e individual (Alarcón, 2014).

Por otro lado, expresan que resulta complicado ejercer dentro de una organización un liderazgo positivo con limitaciones, lo que significa que, cada paso que un líder quiera dar primero debe ser consultado y aprobado por otras personas. Esto contradice rotundamente con el fundamento que lo caracteriza, que es la intuición, acción interconectada con las emociones neurocientíficas (Bonilla, Salinas \& Cavero, 2018). Es importante aclarar, que las emociones no están conectadas directamente con el romanticismo, ni con la parte esotérica; nos referimos a sensaciones que afectan los estadios físicos y mentales que producen reacción y capacidad de respuesta generadoras de impresión y afecto, que logran impactar positiva y efectivamente en la resonancia del grupo de trabajo subordinado (Blanch, Antino \& Rodríguez, 2016).

Para lograr una visión sustentable basado en el liderazgo positivo, de acuerdo con los entrevistados, se necesita de un enfoque que delinee acciones sacrificables y no como una responsabilidad o imposición (Ramos, Franco, \& Pantoja, 2017). El ser humano por lo general tiene respuestas renuentes cuando se le contradice o es mal entendido por cualquier acción. Por lo tanto, las relaciones interpersonales que debe considerar un líder positivo, deben contener información de primera mano cuya relación sea tanto de la organización como por las emociones del personal que maneja (Tipán, 2017).
La estrategia de integración, permite un canal comunicativo efectivo, que desarrolla calidad y excelencia en el desenvolvimiento del trabajo que se tiene que realizar.

Esta perspectiva, conlleva a inferir que el liderazgo positivo requiere de sutileza y discernimiento para lograr que los subordinados entiendan sus responsabilidades. La figura debe ser capaz de dirigir eficazmente y dar la impresión de que entienden, siguen y respetan los propósitos organizacionales. Al mismo tiempo, emana confianza y proyecta conocimiento sobre las acciones de su entorno, dicho de otro modo, que la impresión que causa en todo momento es lo que determinará su nivel de influencia en los seguidores.

¿Qué componente supra puede avalar el buen desempeño de un liderazgo positivo? Sencillamente las organizaciones, quienes se mantienen en la búsqueda constante del punto de equilibrio para poder cumplir con las metas y objetivos gerenciales planteados. En este momento resulta importante acotar, la necesidad de que las empresas comiencen a realizar cambios significativos en materia de dirección corporativa, en otras palabras, es tiempo de ser revolucionarios (no doctrinario) y resilientes; con la intencionalidad de emplear nuevos paradigmas que sean capaces de presentar resultados más efectivos, que promuevan y fortalezcan elementos estratégicos como la competitividad (ventajas), productividad y rentabilidad, que se convierta en una nueva alternativa de negocio donde se puedan incluir a todos los miembros de una organización.

Así emerge la segunda categoría, las organizaciones saludables, consideradas empresas caracterizadas 
enlainversión deesfuerzos colaborativos, sistemáticos e intencionales; con el fin de maximizar el bienestar de los empleados y la productividad, por medio de la generación de puestos de trabajo bien diseñados, ergonómicos, con adecuados ambientes saludables, orientadas al logro y excelencia para concretar oportunidades equitativas y accesibles para el desarrollo de la carrera y el logro estable de un balance positivo relacionado al trabajo en concordancia con la vida privada del trabajador.

Desde este espectro, de acuerdo con los informantes clave, las organizaciones se esfuerzan por mejorar los procesos y resultados de sus empleados, a tal punto de establecer estrategias para tratar en lo posible de mantenerlos estables dentro del sistema laboral. Las entidades saludables abarcan un entorno integrador, lo cual significa, que incluyen prácticas organizacionales eficientes y efectivas que impactan en la salud psicosocial del capital humano. Todo este proceso debe ser capaz de fusionar las metas de la empresa y las necesidades del personal tomando en consideración aspectos emergentes como: estrés ocupacional, seguridad y salud ocupacional, promoción de la salud laboral e incluso la economía.

Adicionalmente, los entrevistados concuerdan en que la necesidad de obtener resultados integradores producto de la realidad laboral, es lo que hace a estas empresas resilientes busquen promover bienestar, compromiso y sentido de pertenencia; considerados elementos de impactos que generan productividad y solvencia operativa, además de mantener una mirada positiva en circunstancias adversas que hacen sustentar los esfuerzos y resultados. Esto conlleva, a realizar consideraciones profundas relacionales, donde las compañías comienzan a planificar sus tareas enmarcadas desde una visión macro, produciendo y diseñando diversos niveles de análisis; que coadyuvan a un mejor desarrollo del negocio.

Entonces, partiendo de que las organizaciones saludables se esfuerzan por desarrollar esquemas basados en la salud y la producción empresarial y resaltando las prácticas organizacionales, se generan tres niveles enfocados bajo estas estructuras: 1) nivel de tarea, rediseña responsabilidades para mejorar la autonomía; 2) nivel del ambiente social, direccionada para ejercer un liderazgo positivo efectivo; y 3) nivel organizacional, cuya relación se armoniza con las prácticas organizacionales para la mejora de la salud, la conciliación trabajo y la familia.

Tomando en consideración, las respuestas de los informantes claves en relación al tema, se encontró la existencia de un ciclo, por la interacción dinámica entre sus elementos, el cual los investigadores han denominado SARF (Siembra-A lo largo de-Frutos), que no es más que el aglutinamiento de procesos, factores, componentes y elementos surgidos de las subcategorizaciones que se focalizan como un sistema, representado en el diagrama 3 : 


\section{Diagrama 3}

\section{Sistema SARF - Siembra-A lo largo de-Frutos.}

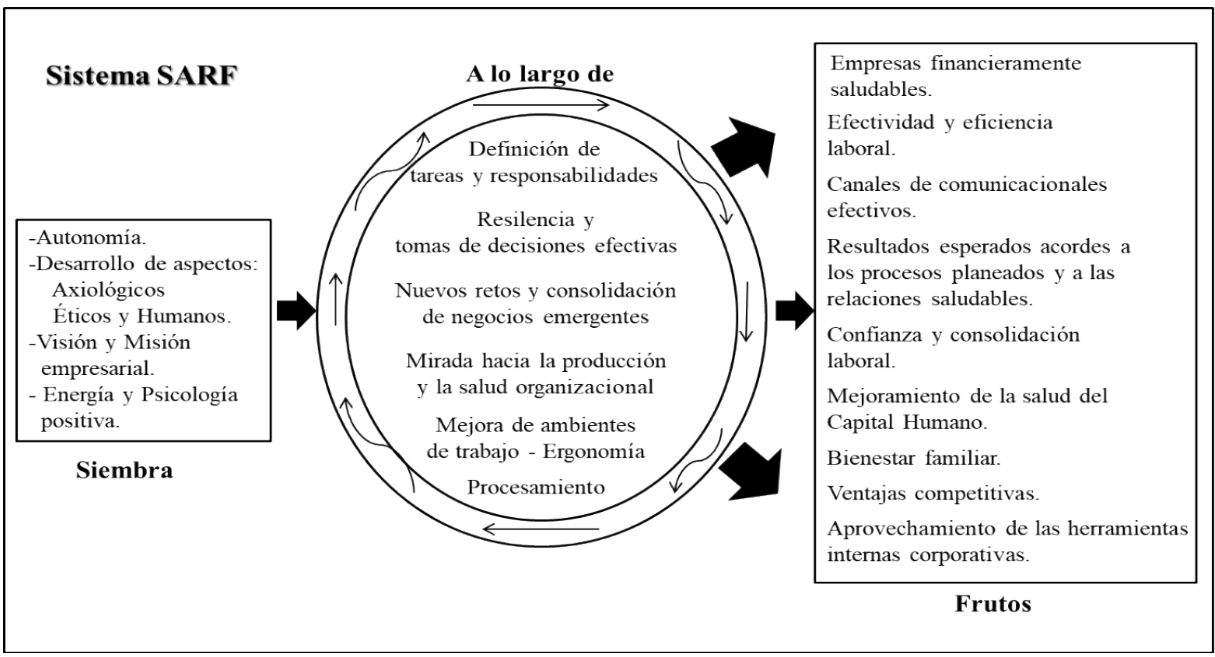

Fuente: Elaboración propia.

En este ciclo, iniciado por la siembra (liderazgo positivo) de elementos como: Autonomía; desarrollo de aspectos axiológicos, éticos y humanos; la declaración clara de la visión y misión del negocio; y la presencia de una energía y psicología positiva conlleva a que a lo largo de la generación de procesos asociados a las actividades regulares de la organización, entre los que se tienen: definición de tareas y responsabilidades; resiliencia y toma de decisiones efectivas; asunción de retos y consolidación de negocios emergentes; la producción y salud organizacional; la mejora del ambiente de trabajo. Tengan como frutos 0 resultados: empresas financieramente saludables; efectividad y eficiencia laboral; canales de comunicación efectivos; mejor salud de los empleados; bienestar familiar; ventajas competitivas.
La coordinación operativa y el nivel de comunicación que debe existir en todos los niveles organizacionales para poder encausar y consolidar este sistema SARF, debe ser altamente confiable, claro, preciso y sobre todo prospectivo ¿Qué se quiere decir con esto? Si se quiere ser innovador $y$ creativo y, a su vez generar resultados competitivos bajo un esquema basado en la coordinación de las actividades desde la salud de los trabajadores, se debe estar preparado para ocasionar un principio de doble reacción (salud financiera y capital humano). Cuando se analiza este principio, se entiende que el funcionamiento operativo que se crea al momento de asociar el liderazgo positivo en organizaciones saludables, origina un efecto multitareas que repercute o impacta al mismo tiempo en el crecimiento o rendimiento financiero 
organizacional.

Finalmente y como tercera categoría, tipo emergente, se tiene la responsabilidad social, la cual fue enriquecedora porque nace del fruto del análisis de la matriz triangular. En ese sentido, sencillamente se blindó desde todos los ángulos tanto las operaciones generales de la empresa como su alcance hacia una conciencia social. Conjugando temas sociales con resultados saludables es prácticamente una combinación que podría ser vista como modelo o referencia organizativa, y una forma de retribuir bienestar al entorno. Para adicionar este elemento, es necesario que las organizaciones saludables generen empatía y orientación hacia el servicio comunitario, paso principal para engendrar compromiso social como una estrategia intrínseca de la gestión empresarial.

\section{Conclusiones}

Las empresas en la búsqueda de asegurar una ventaja competitiva, emplea como herramientas las estrategias comerciales. Sin embargo, derivado por las nuevas disrupciones y avances tecnológicos, en la actualidad las mismas organizaciones tienen la oportunidad de emplear recursos innovadores y competitivos que fortifiquen las actividades desde todos los ámbitos, aprovechando sus recursos incluyendo el capital humano. Este nuevo esquema o impulso corporativo, se puede concentrar mediante la adecuada incursión del liderazgo positivo, que no es más que una estrategia gerencial que sirve para lograr cambios de conducta, bien sea individual o grupal, con el fin de cumplir objetivos y metas de la organización.

El liderazgo positivo además de tener repercusiones axiológicas, éticas y estratégicas, es preponderante en el ciclo del negocio ya que actúa como impulsor de procesos que enriquecen aspectos financieros, productivos y de recursos humanos (salud). El liderazgo positivo, denota valor agregado en las organizaciones saludables, que debido a su término compuesto (salud y organización), conlleva una visión integradora que incluye resultados de un variado número de disciplinas $y$ especialidades, que comprende no solo las perspectivas tradicionales de salud en el lugar de trabajo (estrés ocupacional, seguridad y salud ocupacional y la promoción de la salud laboral), sino también la conducta organizacional, la dirección de recursos humanos y la economía.

Partiendo del objetivo del estudio relacionadas a las implicaciones del liderazgo positivo dentro de organizaciones saludables, es bueno destacar que la investigación condujo a develar la importancia de todo el componente que forma parte de un proceso interesante donde se le da un espacio representativo al capital humano, convirtiéndose en pieza fundamental para que las organizaciones puedan superar y cumplir las expectativas planteadas.

Considerar el liderazgo positivo en las organizaciones saludables, encamina a las empresas a repensar su forma de trabajo tradicional, agregando procesos que puedan contribuir al desarrollo y crecimiento de la salud entre sus trabajadores, el cual incluye hasta adecuados ambientes de trabajo y autonomía para decidir sobre lo mejor para mejorar la productividad empresarial.

A partir de la visión epistemológica de los ejes temáticos, en concordancia 
Sánchez Montero, Ivanna Karina; Ríos Mariño, Marcelo Javier;

Cajas Cajas, Viviana Elizabeth y Tanqueño Colcha, Oscar Paul

Liderazgo positivo en organizaciones saludables

con las respuestas de los informantes claves, se creó un sistema denominado SARF que sirve de guía o modelo para ir reconocimiento de manera sucinta los efectos positivos generados por el empleo de un programa basado en el liderazgo positivo en las organizaciones saludables. Se deja claro, que la incidencia de estos componentes empleados de forma armonizada, ofrece alternativas para la obtención de resultados satisfactorios en los negocios, logrando romper esquemas tradicionales que no permiten que las empresas crezcan, se fortalezcan y sostengan en el mercado donde se desenvuelven.

El liderazgo positivo y sus implicaciones en las prácticas organizacionales saludables, generan un cambio paradigmático beneficioso para aquellas entidades que logren enmarcarse en ella. Quedando comprobado que la unificación entre lo gerencial y lo emocional, apertura brechas comunicacionales que cuando son enfocadas correctamente, producen resultados altamente confiables $y$ beneficiosos, inclusive llegan a crear escenarios estratégicos necesarios para que la alta gerencia pueda tomar decisiones acertadas en el bienestar de todos los involucrados en la organización

\section{Referencias Bibliográficas}

Acosta, H., Torrente, P., Llorens, S., y Salanova, M. (2013). Prácticas organizacionales saludables: Un análisis exploratorio de su impacto relativo sobre el engagement con el trabajo. Psicología y Trabajo Social, 2(1), 107-120. http://www.want.uji.es/ wp-content/uploads/2017/02/2013 Acosta-Torrente-Llorens-Salanova. pdf

Alarcón, M. (2014). Factores claves de los líderes del Siglo 21. Revista Publicando, 1(1), 46-58. https:// revistapublicando.org/revista/index. php/crv/article/view/12

Baño, N. (2019). Sistema de formación para líderes directivos de instituciones educativas [Tesis de posgrado, Universidad Tecnológica Indoamérica]. https://bit.ly/3i9FdYW

Belanger, J., Pierro, A., Kruglanski, A., Vallerand, R., \& Falco, A. (2015). On feeling good at work: the role of regulatory mode and passion in psychological adjustment. Journal of Applied Social Psychology, 45(6), 319-329. https://doi.org/10.1111/ jasp.12298

Benavides, C. (2017). Estilos de liderazgo en las redacciones chilenas: estudio de la autopercepción de los directivos. Cuadernos.info, (41), 123-140. https://doi.org/10.7764/ cdi.41.1133

Blanch, J., Gil, F., Antino, M., \& Rodríguez, A. (2016). Modelos de liderazgo positivo: Marco teórico y líneas de investigación. Papeles Del Psicólogo, 37(3), 170-176. http://www.papelesdelpsicologo.es/ pdf/2772.pdf

Bonilla, D., Macero, R., \& Mora, E. (2018). La importancia de la capacitación del personal administrativo de la Universidad Técnica de Ambato. Revista Conrado, 14(63). https://bit. ly/3kokS52

Bonilla, D., Pozo, C., y Mayorga, V. (2018). Conociendo al consumidor ecuatoriano desde la antropología y el marketing: una perspectiva psicológica gerencial. Polo del Conocimiento, 3(6), 96-109. https:// doi.org/10.23857/pc.v3i6.507

Bonilla, D., Salinas, D., \& Cavero, O. (2018). Modelo de valuación de puestos de trabajo (VPT) basado en el 
método de puntuación. Universidad, Ciencia y Tecnología, 1, 46-59. http://uctunexpo.autanabooks.com/ index.php/uct/article/view/47

Cameron, K. (2013). Practicing Positive Leadership; Tools and Techniques That Create Extraordinary Results, (1ra. Ed.). Berrett-Koehler Publishers, Inc.

Capa, Á., Cruz, A., Tejeda, A., Gutiérrez, L., Llontop, H., y Moreno, A. (2020). Introducción del liderazgo positivo: oportunidades de mejora en el aprendizaje en la educación superior. Revista Internacional de Aprendizaje en la Educación Superior, 7(1), 2937. https://doi.org/10.37467/gkarevedusup.v7.2020

Cedeño, A., Asencio, L., y Villegas, M. (2019). Las estrategias gerenciales como base fundamental para la administración en los negocios. Universidad y Sociedad, 11(5), 191200. https://rus.ucf.edu.cu/index.php/ rus

Chiniara, M., \& Bentein, K. (2016). Linking servant leadership to individual performance: Differentiating the mediating role of autonomy, competence and relatedness need satisfaction. The Leadership Quarterly, 27(1), 124-141. https://doi. org/10.1016/j.leaqua.2015.08.004

Coronel, J., y Marzo, N. (2017). La promoción de la salud: evolución y retos en América Latina. MEDISAN, 21(7), 926-932. http://medisan.sld. cu/index.php/san/article/vie w/1592

David, J., \& Quintero, Z. (2016). Factores influyentes en el bienestar de los individuos en un contexto laboral. PSYCONEX, 8(12), 1-9. https://doi. org/10.15649/2346030X.559

Díaz, M. (2017). El liderazgo positivo como herramienta para generar empresas saludables y felices. $R H$
Saludable, 1. https://rhsaludable. com/el-liderazgo-positivo-comoherramienta-para-generarempresas-saludables-y-felices

Donoso, S., y Benavides, N. (2018). Prácticas de gestión de los equipos directivos de escuelas públicas chilenas. Brasileira de Educacao, 23(1), 1-28. http://dx.doi.org/10.1590/ s1413-24782018230013

Fuster, Y. (2020). Organizaciones saludables: personas e información en sinergia y desarrollo. Brazilian Journal of Information Science, 14(3), e020002. https://doi. org/10.36311/1940-1640.2020. $\underline{\mathrm{v} 14 \mathrm{n} 3.10059}$

Gil, F., Alcover, C., Rico, R., y Sánchez, M. (2011). Nuevas formas de liderazgo en equipos de trabajo. Papeles del Psicólogo, 32(1), 3847. https://dialnet.Unirioja.es/servlet/ articulo?codigo=3406744\%0Ahttp:// www.redalyc.org/articulo. oa?id=77817210005

González, M. (2019). La arquitectura del liderazgo: ¿El liderazgo nace, se hace o se estructura? Debates IESA, 24(2), 13-17. https://bit.ly/3enuusZ

Goodall, A., \& Pogrebna, G. (2014). Expert leaders in a fast-moving environment. Leadership Quarterly, 26(2), 123-142. https://doi. org/10.1016/j.leaqua.2014.07.009

Guerrero, A., Marín, M., y Bonilla, D. (2018). Erp como alternativa de eficiencia en la gestión financiera de las empresas. Revista Lasallista de Investigación, 15(2). https://doi. org/10.22507/rli.v15n2a14

Ñaupas, H., Valdivia, M., Palacios, J., y Romero, H. (2018). Metodología de la investigación cuantitativa $y$ cualitativa y redacción de tesis $\left(5^{\mathrm{a}}\right.$ Ed.). Ediciones de la U. https:// corladancash.com/wp-content/ 
Sánchez Montero, Ivanna Karina; Ríos Mariño, Marcelo Javier;

Cajas Cajas, Viviana Elizabeth y Tanqueño Colcha, Oscar Paul

Liderazgo positivo en organizaciones saludables

uploads/2020/01/Metodologia-de-lainv-cuanti-y-cuali-Humberto-NaupasPaitan.pdf

Organización Internacional del TrabajoOIT (2008). La Declaración de Seúl sobre seguridad y salud en el trabajo. https://www.ilo.org/wcmsp5/ groups/public/@dgreports/@ dcomm/documents/statement/ wcms 095955.pdf

Reese, S. (2020). Taking the learning organization mainstream and beyond the organizational level: An interview with Peter Senge. The Learning Organization 1(23), 1-16. https://doi. org/10.1108/TLO-09-2019-0136

Rico, D. (2016). El modelo de empresa saludable: un modelo posible también para las PYMES. Prevencionar. http://prevencionar.com/2016/02/14/ el-modelo-de-empresa-saludableun-modelo-posible-tambien-paralas-pymes/

Rocha, Á. (2019). Leadership styles and skills developed through game-based learning. Journal of Business Research, Elsevier, 94(c), 360-366. https://doi.org/10.1016/j. ibusres.2018.01.057

Roche, M., Haar, J. M., \& Luthans, F. (2016). The Role of Mindfulness and Psychological Capital on the Well-Being of Leaders. Journal of Occupational Health Psychology, 1-16. http://dx.doi.org/10.1037/ $\underline{\mathrm{a} 0037183}$

Ramos, V., Franco, A., \& Pantoja, O. (2017). Estudio de liderazgo en Ecuador. Encuentro Internacional de Investigadores En Administración. Encuentro Internacional de Investigadores en Administración. Bogotá. https://bit.ly/2UQ1aEe

Romero, F., y Loja, R. (2011). El fortalecimiento de la organización barrial en Ecuador como elemento necesario para el avance del desarrollo social. El caso Loja. Contribuciones a las Ciencias Sociales, 1(1). https://www.eumed. net/rev/cccss/1 3/rflr.html

Salanova, M. (2008). Organizaciones saludables y desarrollo de recursos humanos. Revista de Trabajo y Seguridad Social, 47(303), 179-214. https://www.want.uii.es/ wp-content/ uploads/2017/03/2008 Salanova.pdf

Salanova, M. (2009). Organizaciones saludables, organizaciones resilientes. Gestión Práctica de Riesgos Laborales, 58(1), 1823. https://repositori.uji.es/xmlui/ bitstream/handle/10234/ 232/32403. pdf? sequence $=1$

Salanova, M., Llorens, S., y Martínez, I. (2016). Aportaciones desde la psicología organizacional positiva para desarrollar organizaciones saludables y resilientes. Papeles del Psicólogo, 37(3), 177-184. https:// psycnet.apa.org/record/2016-57 110$\underline{004}$

Silva, M., \& Bonilla, D. (2020). Justicia organizacional y capital psicológico: factores que se relacionan con la satisfacción laboral. Magazine de Las Ciencias: Revista de Investigación e Innovación, 5(7), 16-29. https:// revistas.utb.edu.ec/index.php/ magazine/article/download/838/680/

Sena, C. (2016). Organizaciones saludables [Tesis de posgrado, Universitas Miguel Hernández]. https://dspace.umh.es/ bitstream/11000/3416/1/Sena\%20 de $\% 20$ Haro $\% 2$ C $\% 20$ Carolina $\% 20$ TFM.pdfH.pdf

Solares, J., Peñalver, J., Meneghel, I., y Salanova, M. (2016). Desarrollando equipos empáticos: la influencia de las prácticas organizacionales saludables en la empatía colectiva. Revista Psicología, 6(1), 51-63. 
https://revistas.ucsp.edu.pe/index. php/psicologia/article/view/38/39

Thomas, H. (2017). ¿Hay empresas saludables? Tres maneras de responder a esta pregunta. Revista de los Estudios de Economía $y$ Empresa, 8(1), 59-70. https://comein. uoc.edu/divulgacio/oikonomics/ recursos/documents/08/5 Thomas Oikonomics 8 cast a4.pdf

Tipán, F. (2017). Propuesta de un plan de comunicación interna para la mejora del clima organizacional de la Dirección Distrital de Educación 17D04 CENTRO en el año 2017. [Universidad Central del Ecuador]. http://www.dspace.uce.edu.ec/ handle/25000/9005

Vaca, A. (2019). La importancia de la honestidad y el liderazgo en las organizaciones. ADGHE, 5(1), 20 21. https://www.adghe.com/index. php/revista

Villavicencio, M. (2017). Trabajadores sanos en organizaciones sanas: Análisis sobre las prácticas organizacionales saludables en un grupo empresarial del sector farmacéutico de la ciudad de Guayaquil. [Tesis de posgrado, UEES]. https://repositorio.uees.edu. ec/handle/123456789/1201

Wilson, M. G., Dejoy, D. M., Vandenberg, R. J., Richardson, H. A., \& Mcgrath, A. L. (2004). Work characteristics and employee health and well-being: Test of a model of healthy work organization. Journal of Occupational and Organizational Psychology, 77(4), 565-588. http://dx.doi. org/10.1348/0963179042596522 